\title{
Calculation of Aerothermodynamics for High-Speed Aircraft X-43 Using Computer Code UST3D and UST3D-AUSMPW
}

\author{
P. Silvestrov ${ }^{1}$ and S. Surzhikov ${ }^{2}$ \\ ${ }^{1}$ Tactical Missiles Corporation, \\ Korolev, Moscow district, 141080, Russia \\ ${ }^{2}$ Institute for Problems in Mechanics of the Russian Academy of Sciences, \\ Moscow, 119526, Russia \\ pavelsilvestrov@yandex.ru
}

\begin{abstract}
In this article computer modeling of the aerodynamic coefficients for a high-speed aircraft model, which is analog to $\mathrm{X}-43$ at Mach number $\mathrm{M}=7$ is performed. Calculation was realized using original and modified copyright computer codes (UST3D and UST3D-AUSMPW), which allow modeling the aerothermodynamic characteristics of aircraft with random geometry using three-dimensional unstructured tetrahedral grids. These copyright computer codes implement a model of viscous compressible heat-conducting gas, described by a spatial non-stationary system of Navier-Stokes equations. The theoretical aspects of aerothermodynamics computer modeling for high-speed aircraft are considered. Cross-verification copyright computer codes was implemented using distribution of the aerodynamic parameters, values of aerodynamic characteristics, as well as the time of solution. Both copyright computer codes gives a fairly reliable picture of the distribution for sought-for quantities fields, and also calculate the aerodynamic characteristics with high accuracy relative to each other.
\end{abstract}

Keywords: gas dynamics, mathematic modelling, computational aerodynamics, copyright computer codes, unstructured grids, AUSM scheme, cross verification.

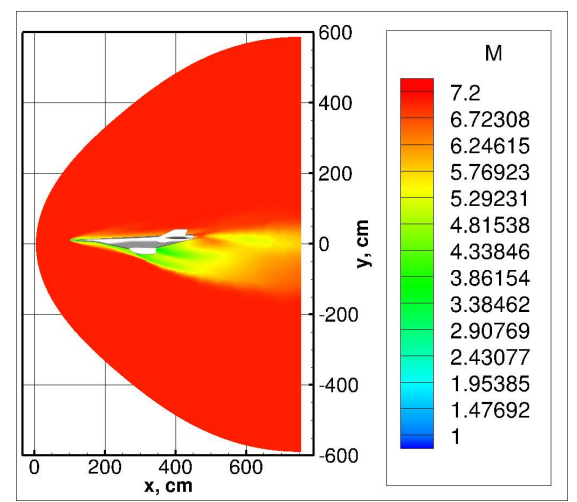

Mach number distribution using UST3D

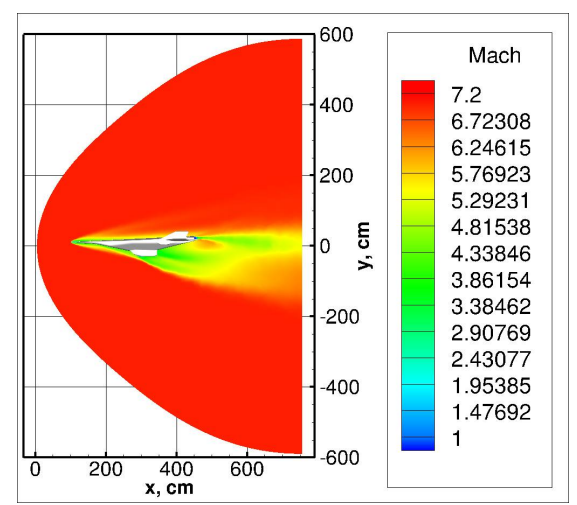

Mach number distribution using UST3D-AUSMPW 


\title{
Расчет аэротермодинамики высокоскоростного летательного аппарата X-43 с использованием компьютерных кодов UST3D и UST3D-AUSMPW
}

\author{
Сильвестров П.В. ${ }^{1}$, Суржиков С.Т. ${ }^{2}$ \\ ${ }^{1}$ Корпорация «Тактическое ракетное вооружение», \\ Россия, Московская обл., Королев, 141080, ул. Ильича, 7 \\ ${ }^{2}$ Институт проблем механики им. А.Ю. Иилинского Российской академии наук, \\ Россия, Москва, 119526, проспект Вернадского, 101-1 \\ pavelsilvestrov@yandex.ru
}

\begin{abstract}
Аннотация
Выполнено компьютерное моделирование аэродинамических коэффициентов модели высокоскоростного летательного аппарата, аналогичного X-43, движущегося с числом Маха $\mathrm{M}=7$. Расчеты проводилось с использованием оригинального и модифицированного авторских компьютерных кодов (UST3D и UST3D-AUSMPW), которые позволяют моделировать аэротермодинамику летательных аппаратов с произвольной геометрией на трехмерных неструктурированных тетраэдральных сетках. В основе данных кодов лежит модель вязкого сжимаемого теплопроводного газа, которая описывается пространственной нестационарной системой уравнений Навье-Стокса. Рассмотрены теоретические аспекты компьютерного моделирования аэротермодинамики высокоскоростных летательных аппаратов. Проведена перекрестная верификация данных компьютерных кодов на основе сравнения распределений аэродинамических параметров, значений аэродинамических характеристик, а также времени установления решения. Показано, что указанные компьютерные коды дают достаточно достоверную картину распределения полей искомых величин, а также рассчитывают аэродинамические характеристики с высокой точностью относительно друг друга.
\end{abstract}

Ключевые слова: газовая динамика, математическое моделирование, вычислительная аэродинамика, авторские компьютерные коды, неструктурированные сетки, схема AUSM, перекрестная верификация.

\section{1. Введение}

Одной из наиболее актуальных проблем разработки летательных аппаратов нового поколения является изучение их гиперзвукового пространственного обтекания.

Высокая стоимость проведения летных и наземных испытаний перспективных изделий повышает роль численного моделирования, так как его применение позволяет существенно снизить число наземных и летных экспериментов, что позволяет существенно снизить стоимость разработки летательных аппаратов. Также ограничение на использование только реальных испытаний накладывает тот факт, что в ходе экспериментов можно измерить лишь отдельные газодинамические величины. При этом в результате расчета возможно получение всех параметров течения $[1,2]$.

Проблеме изучения гиперзвуковой аэродинамики посвящено большое количество работ, например [3, 4]. Задача расчетного анализа особенностей аэротермодинамики летательных аппаратов сложной формы рассмотрена в таких работах, как [5-8] для моделирования на 
неструктурированных сетках и [9] на структурированных сетках. Гиперзвуковая аэродинамика для тел упрощенной формы исследуется в работах $[10,11]$.

В данной статье описаны результаты численного моделирования структуры течения вокруг модели высокоскоростного летательного аппарата, аналогичного X-43. Для этого был использован авторский компьютерный код UST3D в двух модификациях, реализующих разные подходы к расчету потока на границах ячеек объемной неструктурированной тетраэдральной сетки расчетной области. В основе данных компьютерных кодов лежит численное решение полных уравнений Навье - Стокса для ламинарного режима течения.

\section{2. Математическая постановка, метод решения задачи и принятые допущения}

Пространственное обтекание модели летательного аппарата описывается трехмерной нестационарной системой уравнений Навье - Стокса, которая записана в векторном виде [7]

$$
\frac{\partial \mathbf{w}}{\partial t}+\operatorname{div} \mathbf{F}=\operatorname{div} \mathbf{G}
$$

В данном выражении

$$
\begin{gathered}
\mathbf{w}=\left(\begin{array}{c}
\rho \\
\rho u \\
\rho v \\
\rho w \\
\rho E
\end{array}\right), \quad \mathbf{F}=\left(\begin{array}{c}
\rho u \\
\rho u^{2}+p \\
\rho u v \\
\rho u w \\
\rho u E+p u
\end{array}\right)+\left(\begin{array}{c}
\rho v \\
\rho u v \\
\rho v^{2}+p \\
\rho v w \\
\rho v E+p v
\end{array}\right)+\left(\begin{array}{c}
\rho w \\
\rho u w \\
\rho v w \\
\rho w^{2}+p \\
\rho w E+p w
\end{array}\right), \\
\mathbf{G}=\left(\begin{array}{c}
0 \\
\tau_{x x} \\
\tau_{y x} \\
\tau_{z x} \\
\tau_{z y} \\
u \tau_{x x}+v \tau_{y x}+w \tau_{z x}-q_{x}
\end{array}\right)+\left(\begin{array}{c}
0 \\
\tau_{x z} \\
\tau_{y z} \\
\tau_{z z} \\
u \tau_{x y}+v \tau_{y y}+w \tau_{z y}-q_{y}
\end{array}\right)
\end{gathered}
$$

где $\mathbf{w}$ - вектор консервативных переменных; $\mathbf{F}$ - вектор конвективного потока; $\mathbf{G}$ - вектор вязкого потока; $\rho$ - плотность; $p$ - давление; $u, v, w$ - компоненты вектора скорости; $E$ удельная полная энергия газа; $\tau_{i, j}-$ компонент тензора вязких напряжений $\boldsymbol{\tau} ; q_{x}, q_{y}, q_{z}-$ компоненты вектора теплового потока.

Тензор вязких напряжений записывается в виде

$$
\boldsymbol{\tau}=\mu\left(\begin{array}{ccc}
\frac{4}{3} \frac{\partial u}{\partial x}-\frac{2}{3} \frac{\partial v}{\partial y}-\frac{2}{3} \frac{\partial w}{\partial z} & \frac{\partial v}{\partial x}+\frac{\partial u}{\partial y} & \frac{\partial w}{\partial x}+\frac{\partial u}{\partial z} \\
\frac{\partial v}{\partial x}+\frac{\partial u}{\partial y} & \frac{4}{3} \frac{\partial v}{\partial y}-\frac{2}{3} \frac{\partial u}{\partial x}-\frac{2}{3} \frac{\partial w}{\partial z} & \frac{\partial w}{\partial y}+\frac{\partial v}{\partial x} \\
\frac{\partial w}{\partial x}+\frac{\partial u}{\partial z} & \frac{\partial w}{\partial y}+\frac{\partial v}{\partial x} & \frac{4}{3} \frac{\partial w}{\partial z}-\frac{2}{3} \frac{\partial u}{\partial x}-\frac{2}{3} \frac{\partial v}{\partial y}
\end{array}\right)
$$

Здесь $\mu-$ коэффициент динамической вязкости.

В качестве замыкающих соотношений используются:

$p=(\gamma-1) \rho U-$ уравнение состояния совершенного газа;

$U=c_{V} T-$ калорическое уравнение состояния; 


$$
\begin{aligned}
& E=U+\frac{u^{2}+v^{2}+w^{2}}{2}-\text { выражение для удельной полной энергии; } \\
& q=-\lambda \nabla T-\text { закон Фурье. }
\end{aligned}
$$

Здесь $U$ - удельная внутренняя энергия газа; $c_{V}$ - удельная теплоемкость газового потока; $T-$ температура; $\lambda$ - коэффициент теплопроводности.

Постановка граничных условий осуществлялась следующим образом. На входной границе расчетной области $G_{\text {in }}$ используются условия в набегающем потоке газа

$$
\begin{gathered}
\left.p\right|_{G_{\text {in }}}=p_{\infty} ;\left.\rho\right|_{G_{\text {in }}}=\rho_{\infty} ;\left.u\right|_{G_{\text {in }}}=u_{\infty} ;\left.v\right|_{G_{\text {in }}}=v_{\infty} ;\left.w\right|_{G_{\text {in }}}=w_{\infty} ; \\
\left.E\right|_{G_{\text {in }}}=E_{\infty}=U+\frac{1}{2}\left(u^{2}+v^{2}+w^{2}\right),
\end{gathered}
$$

где $p_{\infty}, \rho_{\infty}, u_{\infty}, v_{\infty}, w_{\infty}$ - параметры невозмущенного потока.

При вытекании газа на выходе из расчетной области $G_{\text {out }}$ применяются условия открытой границы

$$
\left|\frac{\partial \rho}{\partial \mathrm{n}}\right|_{G_{\text {out }}}=0 ;\left|\frac{\partial u}{\partial \mathrm{n}}\right|_{G_{\text {out }}}=0 ;\left|\frac{\partial v}{\partial \mathrm{n}}\right|_{G_{\text {out }}}=0 ;\left|\frac{\partial w}{\partial \mathrm{n}}\right|_{G_{\text {out }}}=0 ;\left|\frac{\partial E}{\partial \mathrm{n}}\right|_{G_{\text {out }}}=0,
$$

где $n$ - вектор единичной нормали к грани. Во всех расчетах в выходном сечении расчетной области наблюдались сверхзвуковые скорости.

На твердой стенке $G_{\text {wall }}$ ставятся условия прилипания для скорости, условия адиабатичности или изотермичности для температуры. Для энергии используются условия теплоизолированной или охлаждаемой стенки

$$
\left.\frac{\partial p}{\partial \mathrm{n}}\right|_{G_{\text {wall }}}=0 ;\left.u\right|_{G_{\text {wall }}}=0 ;\left.v\right|_{G_{\text {wall }}}=0 ;\left.w\right|_{G_{\text {wall }}}=0 ;\left.\frac{\partial T}{\partial \mathrm{n}}\right|_{G_{\text {wall }}}=0 \text { или }\left.T\right|_{G_{\text {wall }}}=T_{w}
$$

В плоскости симметрии $G_{\text {symm }}$ применяется условие симметрии потока.

При этом на тетраэдральной расчетной сетке с низкой степенью подробности использование граничных условий прилипания для твердой стенки не обеспечивает строгое решение уравнений Навье-Стокса. В данном случае постановка граничных условий на стенке применяется для стабилизации численного решения. В работе [12] показано, что использование граничных условий скольжения и системы уравнений Эйлера в аналогичной постановке задачи позволяет получить схожие значения аэродинамических коэффициентов. Также следует отметить, что при проведении расчетов на таких сетках практически невозможно получить адекватное распределение тепловых потоков.

В данном исследовании при моделирования пространственного обтекания поверхности ГЛА сложной формы реализуется метод расщеплении исходной системы уравнений по физическим процессам $[5,13]$.

Решение задачи, если оно существует, получается в результате установления. В данном методе не используется выделение поверхностей разрыва. Система уравнений для модели вязкого сжимаемого теплопроводного газа записывается в виде законов сохранения, что обеспечивает достаточно высокую точность вычислений, т.к. законы сохранения должны выполняться как для отдельных ячеек, так и для всей расчетной области. Однако, значения потоковых величин на границах расчетных ячеек с использованием данного подхода могут вычисляться по-разному. В компьютерном кодеUST3Dдля определения потока массы через границы расчетных ячеек используется метод донорных ячеек [14]. В модифицированной версии компьютерного кода (UST3D-AUSMPW) для этого предлагается использовать схему AUSM PW[15], относящуюся к семейству AUSM (AdvectionUpstreamSplittingMethod) алго- 
ритмов [6]. Главной особенностью AUSMPW является устранение численных неустойчивостей, наблюдаемых в AUSM + в областях сильных возмущений, путем ввода весовых функций, зависящих от давления.

\section{3. Результаты расчетов}

Расчет газодинамических параметров и аэродинамических характеристик проводился на примере высокоскоростного летательного аппарата (ВЛА) X-43. В качестве прообраза выбранной модели были использованы эскизы, взятые из [16-17]. Характерные параметры летательного аппарата: длина - 3.66 м, взлетная масса 1300 кг, запас топлива 1.46 кг [18].

В качестве результатов моделирования аэродинамики приводятся двумерные распределения различных газодинамических параметров в плоскости симметрии $(Z=0)$.

В качестве аэродинамических характеристик использовались коэффициенты подъемной силы и лобового сопротивления, а также аэродинамического качества.

Коэффициент подъемной силы $c_{L}$ определялся по формуле

$$
c_{L}=\frac{F_{y}}{q_{\infty} S_{x a p}},
$$

где $F_{y}$ - подъемная сила; $S_{x a p}$ - характерная площадь.

Коэффициент лобового сопротивления $c_{D}$ определялся по формуле

$$
c_{D}=\frac{F_{x}}{q_{\infty} S_{x a p}},
$$

где $F_{x}$ - сила лобового сопротивления.

Под аэродинамическим качеством понимается их отношение

$$
K=\frac{c_{L}}{c_{D}}
$$

Исходные данные, использованные при проведении расчетов с использованием указанных авторских компьютерных кодов приведены в табл. 1.

\section{Таблица 1}

Исходные данные для расчета и свойства набегающего потока

\begin{tabular}{|l|c|}
\hline \multicolumn{1}{|c|}{ Параметр } & Значение \\
\hline Число Маха & 7 \\
\hline Угол атаки, град & 2 \\
\hline Давление набегающего потока, Па & 1410 \\
\hline Температура набегающего потока, $\mathrm{K}$ & 227 \\
\hline Число элементов сетки & 2013902 \\
\hline$S_{\text {ref }}, \mathrm{m}^{2}$ & 0.40538 \\
\hline
\end{tabular}

Построенная в рамках исследования неструктурированная тетраэдральная сетка расчетной области показана на рис. 1.

В табл. 2 приведены значения аэродинамических характеристик, полученные с использованием авторских компьютерных кодов UST3D и UST3D-AUSMPW. 


\section{Таблийа 2}

\section{Сравнение результатов расчетов аэродинамических} коэффициентов

\begin{tabular}{|c|c|c|c|c|c|c|}
\hline & \multicolumn{3}{|c|}{ UST3D } & \multicolumn{3}{c|}{ UST3D-AUSMPW } \\
\hline & $c_{L}$ & $c_{D}$ & $K$ & $c_{L}$ & $c_{D}$ & $K$ \\
\hline$\alpha=2^{\circ}$ & 0.266 & 0.157 & 1.6943 & 0.287 & 0.172 & 1.6686 \\
\hline
\end{tabular}

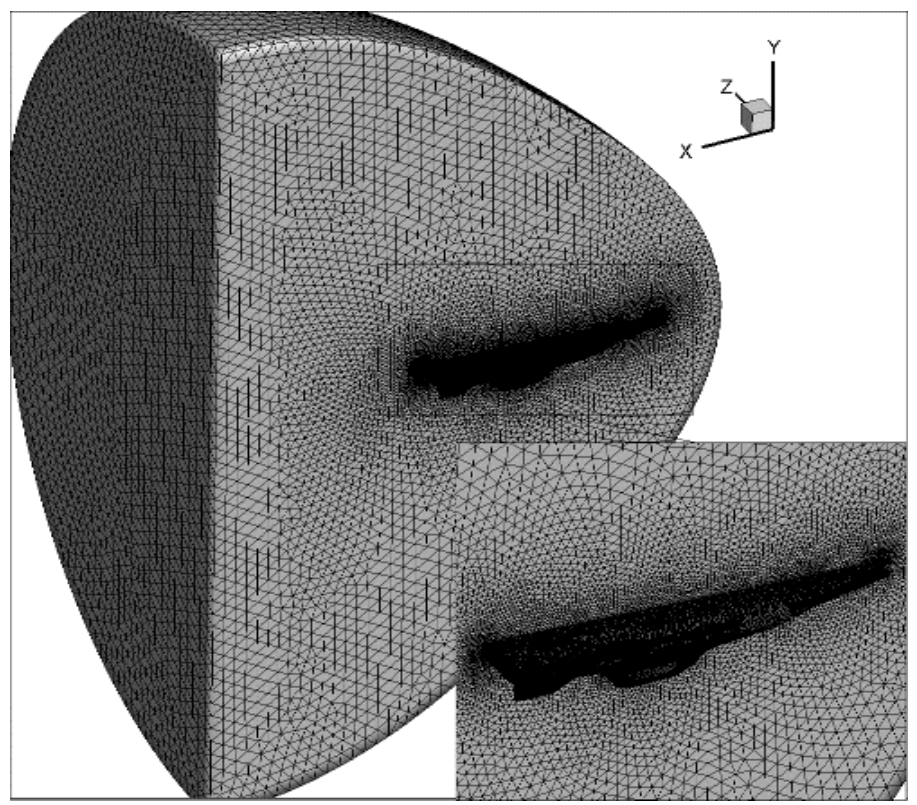

Рис. 1. Неструктурированная тетраэдральная сетка и расчетная область

Двумерное распределение числа Маха, температуры и давления приведено на рис. 2-4. Из рисунков видно, что структура ударных волн, образующихся в носовой части аппарата и перед входом в воздухозаборник, полученная с использованием двух компьютерных кодов, практически не отличается.

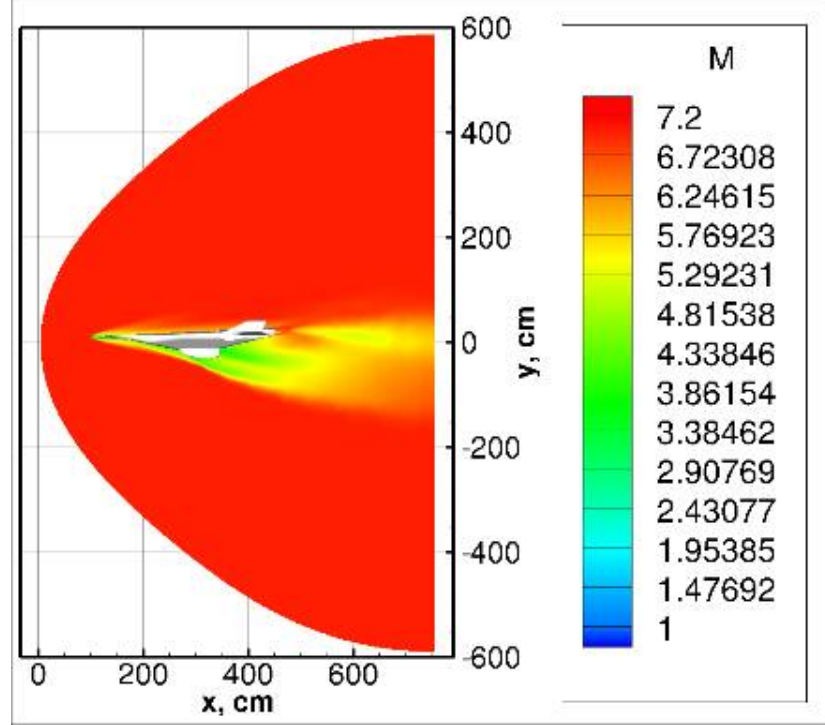

a)

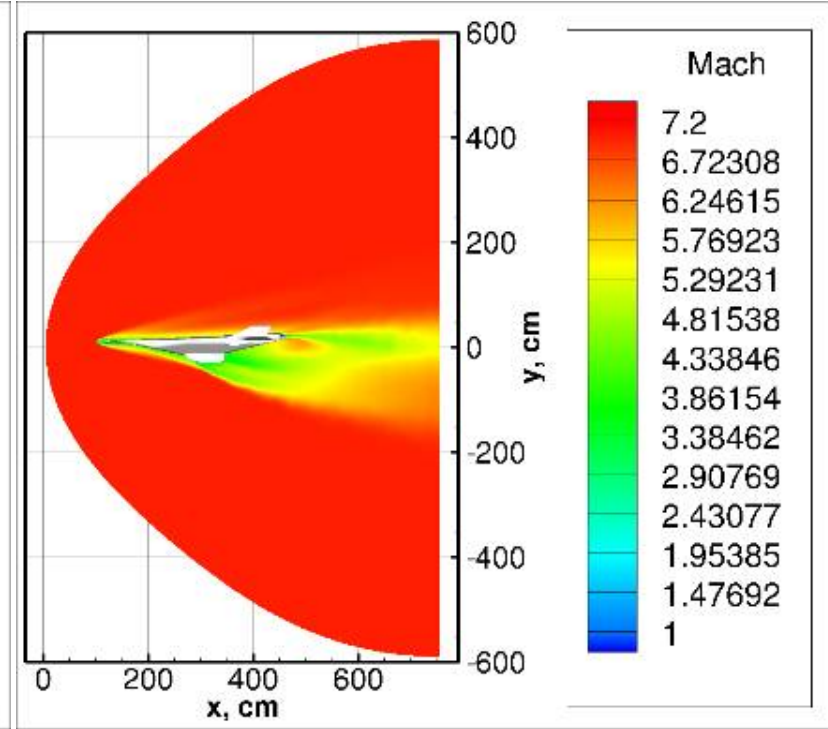

б)

Рис. 2. Двумерное распределение числа Маха при $\alpha=2^{\circ}$ с использованием авторского компьютерного кода: a) UST3D; б) UST3D-AUSMPW 
При этом, как видно из рис. 2 и 4 распределение числа Маха и температуры в отрывной зоне течения в указанных расчетах существенное отличаются - с использованием кода UST3D-AUSMPW диапазон числа Маха и температуры в отрывной зоне выше, чем с использованием кода UST3D.

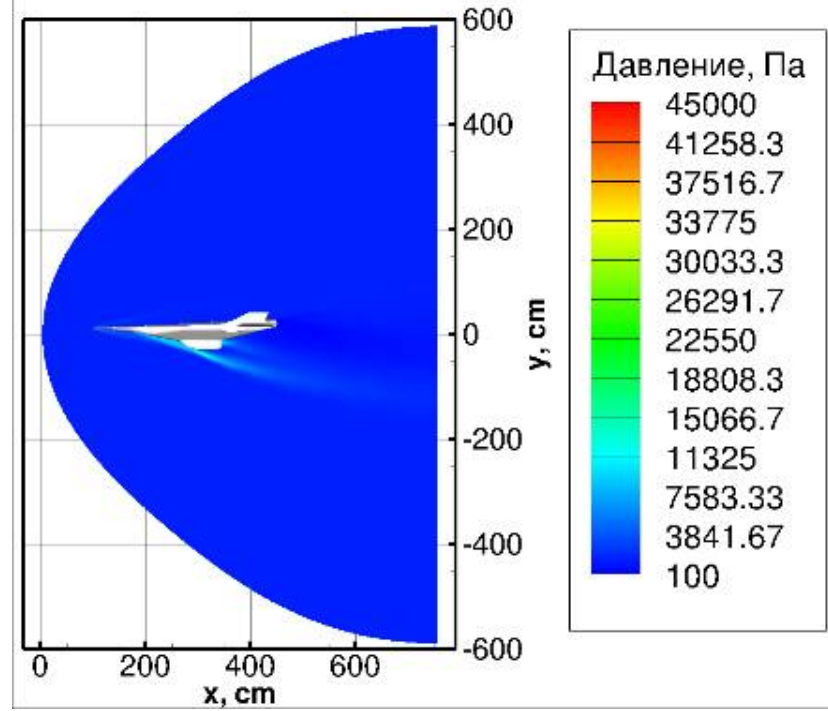

a)

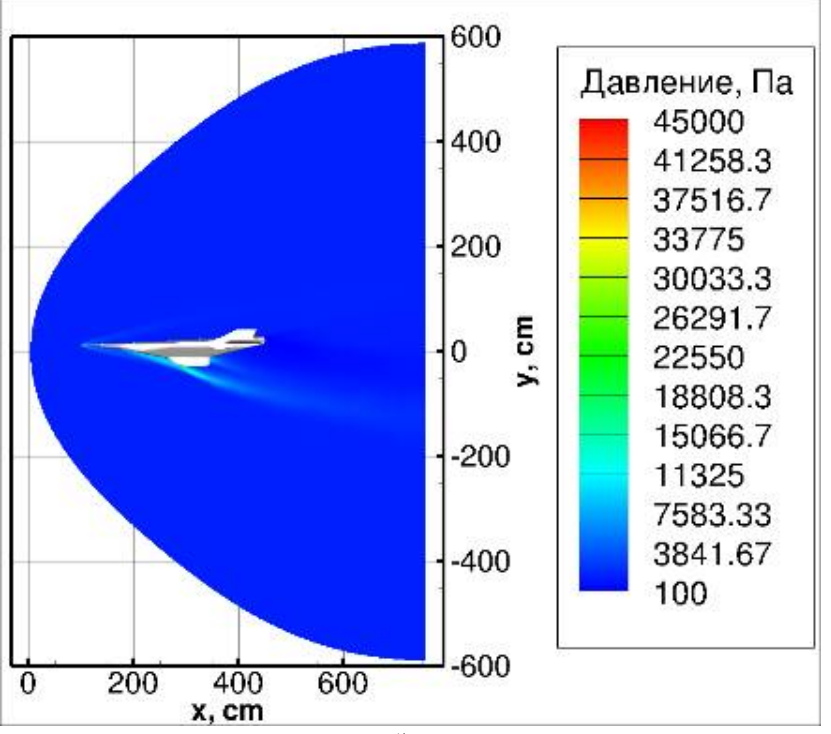

б)

Рис. 3. Двумерное распределение давления (в Па) при $\alpha=2^{\circ}$ с использованием авторского компьютерного кода: $a$ ) UST3D; б) UST3D-AUSMPW

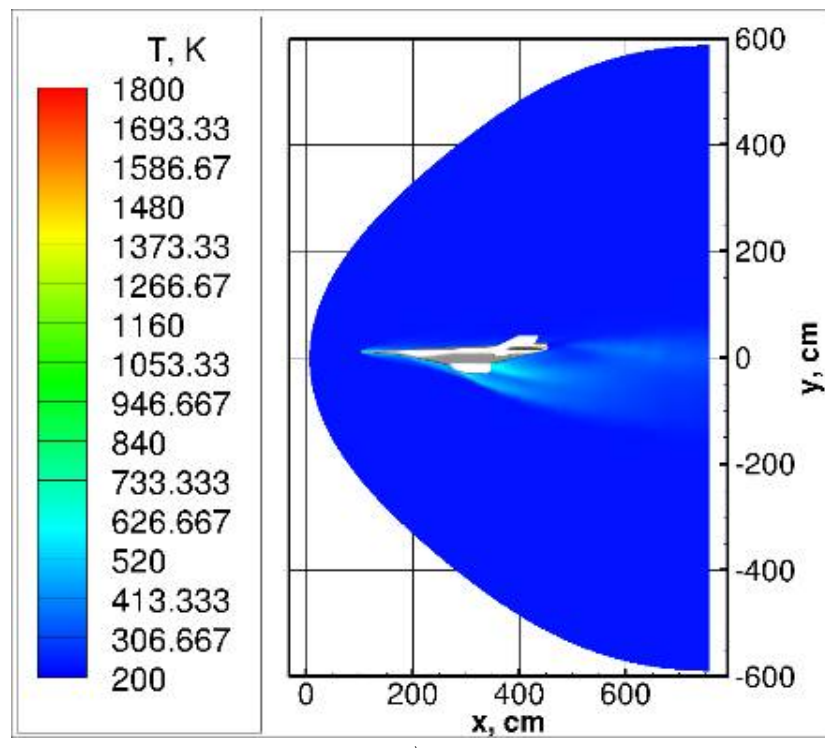

a)

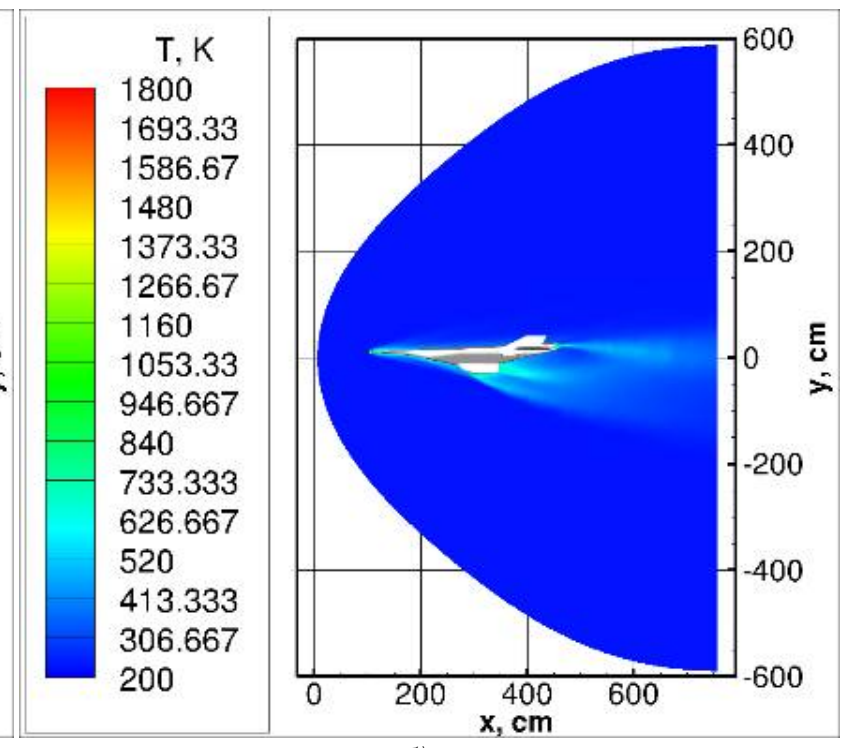

б)

Рис. 4. Двумерное распределение температуры (в K) при $\alpha=2^{\circ}$ с использованием авторского компьютерного кода: a) UST3D; б) UST3D-AUSMPW

Также было проведено сравнение авторских компьютерных кодов UST3D и UST3DAUSMPW по времени расчета аэродинамических характеристик (табл. 3). Как видно из таблицы, расчет с использованием UST3D-AUSMPW требует меньшего числа шагов для установления результатов, но при этом продолжительность расчета каждого шага занимает большее количество времени. Таким образом, при увеличении подробности расчетной сетки эффектность использования модифицированного кода по времени будет снижаться. 
Таблица 3

Сходимость результатов компьютерного моделирования по времени

\begin{tabular}{|l|c|c|}
\hline \multicolumn{1}{|c|}{ Параметр } & UST3D & UST3D-AUSMPW \\
\hline Реальное время, с & 454000 & 90000 \\
\hline Количество шагов & 310000 & 30000 \\
\hline Время расчета одного шага, с & 1.46 & 3 \\
\hline
\end{tabular}

\section{4. Заключение}

Результаты расчетов аэротермодинамики модели, аналогичной ВЛА X-43 показали, что использование авторских компьютерных кодов UST3D и UST3D-AUSMPW дает достаточно достоверную картину распределения полей газодинамических параметров, а также схожие значения аэродинамических характеристик. Имеющиеся различия и погрешности могут быть связаны с недостаточной подробностью расчетной сетки, а также особенностями численного моделирования в областях аэродинамической тени, реализованными в указанных кодах. Следует заметить, что несмотря на низкую подробность расчетной сетки, результаты получаются схожими.

Анализ времени установления расчетов с использованием двух авторских компьютерных кодов по времени показал, что модифицированный компьютерный код позволяет осуществлять расчет за меньшее количество шагов, но при этом каждый шаг занимает большее количество времени.

Работа выполнена по теме государственного задания (№ госрегистрации АAАA-A20120011690135-5) и при поддержке гранта РФФИ № 19-01-00515.

\section{Литература}

1. Schmisseur J.D. A HypersonicsInto the 21st Century: A Perspective on AFOSR-Sponsored Research in Aerothermodynamics in 43rd AIAA Fluid Dynamics Conference. SanDiego, CA. June 24-27, 2013. DOI: 10.2514/6.2013-2606.

2. Bertin J. Hypersonic Aerothermodynamics. AIAA Education Series, 1994. 627 p.

3. Лунев В. В. Гиперзвуковая аэродинамика // М.: Машиностроение, 1975, 328 с.

4. Anderson J. Hypersonic and High-Temperature Gas Dynamics Second Edition // AIAA Education Series, 2006.

5. елезнякова А.Л., Суржиков С.Т. Применение метода расщепления по физическим процессам для расчета гиперзвукового обтекания пространственной модели летательного аппарата сложной формы // ТВT, 2013, т. 51, № 6, C. 897-911. DOI: 10.7868/S0040364413050232.

6. Железнякова А.Л., Суржиков С.Т. Расчет гиперзвукового обтекания тел сложной формы на неструктурированных тетраэдральных сетках с использованием схемы AUSM // TBT, 2014, Т. 52, № 2, c. 283-293. DOI: 10.7868/S0040364414020215.

7. Surzhikov S.T. Validation of computational code UST3D by the example of experimental aerodynamic data. Journal of Physics: Conference Series, 2017, vol. 815, p. 012023. DOI: 10.1088/17426596/815/1/012023.

8. Яцухно Д.С. Суржиков С.Т. Метод расщепления по физическим процессам в задаче моделирования обтекания перспективного высокоскоростного летательного аппарата // Вестник МГТУ им. Н.Э. Баумана. Сер. Машиностроение. 2018. № 1. С. 20-33. DOI: 10.18698/0236-3941-20181-20-33.

9. Суржиков С.Т. Компьютерная аэрофизика спускаемых космических аппаратов. Двухмерные модели // М.: Физматлит, 2018, 544 с. 
10. Суржиков С.Т. Аэрофизика гиперзвукового потока у поверхности спускаемого космического аппарата на высотах менее 60 км // Вестник МГТУ им. Н.Э. Баумана. Серия Естественные науки, 2016, № 5 (68), С. 33-45.

11. Забарко Д.А., Котенев В.П. Численное исследование ламинарных течений вязкого химически реагирующего газа около затупленных тел // Вестник МГТУ им. Н.Э. Баумана. Серия Естественные науки, 2006, № 1 (20), С. 77-95.

12. Kryuchkova A.S. Development and testing of non-viscid solver based on UST3D programming code. Journal of Physics: Conference Series, 2019, vol. 1250, p. 012009. DOI: 10.1088/17426596/1250/1/012009.

13. Белоцерковский О.М., Давыдов Ю.М. Метод крупных частиц в газовой динамике // М.: Наука, 1982, $391 \mathrm{c}$.

14. Roache P. Computational Fluid Dynamics, Hermosa Publishers, P. 434.

15. Kim K., Kim C. and Rho O.-H. Methods for the Accurate Computations of Hypersonic Flows I. AUSMPW+ Scheme. Journal of Computational Physics, 2011, vol. 174, no. 1, pp. 38-80.

16. Reubush D.E., Nguyen L.T., Rausch V.L. Review of X-43A return to flight activities and current status // AIAA 2003-7085. 2003. 12 p.

17. Engelund W.C., Holland S.D, Cockrell C.E. et all. Propulsion system airframe integration issues and aerodynamic database development for the Hyper - X flight research vehicle // ISOABE 99-7215. 1999: $12 \mathrm{p}$.

18. www.nasa.gov/missions/research/x43-main.html

19. Андерсон Д., Таннехилл Дж., Плетчер Р. Вычислительная гидромеханика и теплообмен В 2 томах // М.: Мир, 1990, 728 с.

\section{References}

1. Schmisseur J.D. A Hypersonics into the 21st Century: A Perspective on AFOSR-Sponsored Research in Aerothermodynamics in 43rd AIAA Fluid Dynamics Conference. San Diego, CA. June 24-27, 2013. DOI: $10.2514 / 6.2013-2606$.

2. Bertin J. Hypersonic Aerothermodynamics. AIAA Education Series, 1994. 627 p.

3. Lunev V.V. Hypersonic aerodynamics. M.: Mechanicalengineering, 1975, P. 328.

4. Anderson J. Hypersonic and High-Temperature Gas Dynamics Second Edition // AIAA Education Series, 2006.

5. Zheleznyakova A.L., Surzhikov S.T. Application of the method of splitting by physical processes for the computation of a hypersonic flow over an aircraft model of complex configuration. High Temperature, 2013, vol. 51, № 6, pp. 816-829. DOI: 10.1134/S0018151X13050234.

6. Zheleznyakova A.L., Surzhikov S.T. Calculation of a hypersonic flow over bodies of complex configuration on unstructured tetrahedral meshes using the AUSM scheme High Temperature,2014, vol. 52, № 2, pp. 271-281. DOI: 10.1134/S0018151X14020217.

7. Surzhikov S.T. Validation of computational code UST3D by the example of experimental aerodynamic data. Journal of Physics: Conference Series, 2017, vol. 815, p. 012023. DOI: 10.1088/17426596/815/1/012023.

8. Yatsukhno D.S., Surzhikov S.T. Method for Splitting into Physical Processes in Task of the Flow over a Perspective High-Speed Vehicle Modelling. Vestn. Mosk. Gos. Tekh. Univ. im. N.E. Baumana, Mashinostr. [Herald of the Bauman Moscow State Tech. Univ., Mech. Eng.], 2018, no. 1, pp. 20-33. DOI: 10.18698/0236-3941-2018-1-20-33.

9. Surzhikov S.T. Computer aerophysics of descent spacecraft. Two-dimensional models. M.: Fizmatlit, 2018, P. 544. 
10. Surzhikov S.T. Aerophysics of the hypersonic air flow above surface of space vehicle at altitudes of less than $60 \mathrm{~km}$ Vestn. Mosk. Gos. Tekh. Univ. im. N.E. Baumana, Estestv. Nauki. [Herald of the Bauman Moscow State Tech. Univ., Natural Sciences. Eng.], 2016, no. 5 (68), pp. 33-45.

11. Zabarko D.A., Kotenev V.P. Numerical study of laminar flows of a viscous chemically reacting gas near blunt bodies Vestn. Mosk. Gos. Tekh. Univ. im. N.E. Baumana, Estestv. Nauki. [Herald of the Bauman Moscow State Tech. Univ., Natural Sciences. Eng.], 2006, no. 1 (20), pp. 77-95.

12. Kryuchkova A.S. Development and testing of non-viscid solver based on UST3D programming code. Journal of Physics: Conference Series, 2019, vol. 1250, p. 012009. DOI: 10.1088/1742$6596 / 1250 / 1 / 012009$.

13. Belotserkovsky O.M., Davydov Y.M. The method of large particles in gas dynamics. M.: Science, 1982, P. 391.

14. Roache P. Computational Fluid Dynamics, Hermosa Publishers, P. 434.

15. Kim K., Kim C. and Rho O.-H. Methods for the Accurate Computations of Hypersonic Flows I. AUSMPW+ Scheme. Journal of Computational Physics, 2011, vol. 174, no. 1, pp. 38-80.

16. Reubush D.E., Nguyen L.T., Rausch V.L. Review of X-43A return to flight activities and current status // AIAA 2003-7085. 2003. 12 p.

17. Engelund W.C., Holland S.D, Cockrell C.E., et all. Propulsion system airframe integration issues and aerodynamic database development for the Hyper - X flight research vehicle // ISOABE 99-7215. 1999: $12 \mathrm{p}$.

18. www.nasa.gov/missions/research/x43-main.html

19. Anderson D., Tannehill J., Pletcher R. Computational fluid mechanics and heat transfer. 3rd Edition // CRC Press, 2012, P. 774.

Статья поступила в редакцию 4 марта 2020 г. 